\title{
Effect of the initial stress and rotation on free vibrations in transversely isotropic human long dry bone
}

\author{
S. R. Mahmoud, M. Marin and K.S.Al-Basyouni
}

\begin{abstract}
The object of the present paper is to study the influence of the initial stress and rotation on wave propagation of harmonic waves in a human long dry bone as transversely isotropic material, subject to the boundary conditions that the outer and inner surfaces are traction free. The equations of elastodynamic are solved in terms of displacements. The natural frequency of the plane vibrations in the case of harmonic vibrations has been obtained. The frequencies and the phase velocity are calculated numerically, the effects of the initial stress and rotation are discussed. Comparisons are made with the result in the absence of rotation and initial stress.
\end{abstract}

\section{Introduction}

Application of the poroelastic materials in medicinal fields such as cardiovascular, dental and orthopedics is well known. The investigation of wave propagation over a continuous media has very importance application in the field of engineering, medicine and in bioengineering. The dry bone is piezoelectric in the classical sense [1-2], i.e. mechanical stress results in electric polarization (the indirect effect); and an applied electric field causes strain (the converse effect). Since that time, many others have confirmed the capacity of bones to

Key Words: Bones, Free Vibrations, Initial Stress, Rotation, Transversely Isotropic

2010 Mathematics Subject Classification: Primary 74B05

Received: 25 April, 2014.

Revised: 29 May, 2014.

Accepted: 29 June, 2014. 
EFFECT OF THE INITIAL STRESS AND ROTATION ON FREE VIBRATIONS IN TRANSVERSELY ISOTROPIC HUMAN LONG DRY BONE

produce piezoelectric potentials [3]. Electrical properties of bone are relevant not only as an hypothesized feedback mechanism for bone adaptation and remodelling, but also in the context of external electrical stimulation of bone in order to aid it's healing and repair [4]

In orthopedics propagation of wave over bone is used in monitoring the rate of fracture heating, there are two types of osseous tissue such as trabecular or cancellous and cortical or compact bone, which are of different materials, with respect to their mechanical behavior, in macroscopic terms the porosity percentage in the compact bone is $3-5 \%$, where as in the cancellous or trabecular the porosity percentage is up to $90 \%$ [1].

Mahmoud $[1,5,6]$ investigated the wave propagation under effects of initial stress, rotation and magnetic field in cylindrical poroelastic bones, a granular medium and porous medium. Theoretical analyses of bone piezoelectricity may be relevant to the issue of bone remodeling. Recent, thorough, studies have explored electromechanical effects in wet and dry bone [7-8]. They suggest that two different mechanisms are responsible for these effects: classical piezoelectricity mainly due to the molecular asymmetry of collagen in dry bone, and streaming potentials found in moist or living bone, and generated by the flow of a liquid across charged surfaces. The second mechanism has been argued by dielectric measurements and has suggested that the electromechanical effect in wet (fluid saturated) bone is not due to a piezoelectric effect [9]. Abd-Alla and Mahmoud [10,11] solved magneto-thermoelastic problem in rotating non-homogeneous orthotropic hollow cylindrical under the hyperbolic heat conduction model, and investigated analytical solution of wave propagation in non-homogeneous orthotropic rotating elastic media. Abd-Alla et al. [12] studied propagation of S-wave in a non-homogeneous anisotropic incompressible and initially stressed medium under influence of gravity field. Honarvarla et al.[13]. Ding et al.[14] studied the elasticity of transversely isotropic materials. Chen et al.[15-16] investigated the free vibration and general solution of nonhomogeneous transversely isotropic magneto-electroelastic hollow cylinders. Abd-Alla et al. [17-18] studied problem of transient coupled thermoelasticity of an annular fin and problem of radial vibrations in nonhomogeneity isotropic cylinder under influence of initial stress and magnetic field. Mofakhami et al.[19] studied the finite cylinder vibrations with different end boundary conditions. Abd-Alla et al.[20-21] studied effect of the rotation, magnetic field and initial stress on peristaltic motion of micropolar fluid, and investigated effect of the rotation on a non-homogeneous infinite cylinder of orthotropic.

For the boundary value problem considered in the context of dipolar bodies with stretch, in the paper [23], the authors use some results from the theory of semigroups of the linear operators in order to prove the existence 
EFFECT OF THE INITIAL STRESS AND ROTATION ON FREE VIBRATIONS IN TRANSVERSELY ISOTROPIC HUMAN LONG DRY BONE

and uniqueness of a weak solution, for the initial boundary value problem of a porous thermoelastic body, the authors analyze the temporal behaviour of the solutions [36]. In the paper [24] the problem of reflection and transmission of plane waves at an imperfect boundary between two thermally conducting micropolar elastic solid half spaces with two temperature is investigated. In the paper [37] the authors establish some existence results of the problem at resonance under some appropriate conditions.

In this paper, the equations of elastodynamics for transversely isotropic material under effect of the inatial stress and rotation are solved in terms of displacement potentials. Also, this paper is concerned with the determination of the phase velocity and the eigenvalues of the natural frequency of the plane vibrations of bones under effect of the inatial stress and rotation for different boundary conditions in the cases of harmonic vibrations. The numerical results of the frequency equation are discussed in detail for transversely isotropic material and the effect of inatial stress and rotation for different cases is indicated by figures.

\section{Formulation of the problem}

Let a transversely isotropic long bone as hollow cylinder of inner radius $a$ and outer radius $b$ Taking the cylindrical polar coordinates $(r, \theta, z)$ such that the z-axis pointing vertically upward along the axis of the bone.

The equations of the elastodynamic in rotating medium in present of inatial stress as:

$$
\begin{gathered}
\frac{\partial \sigma_{r r}}{\partial r}+\frac{\partial \tau_{r z}}{\partial z}+\frac{1}{r}\left(\sigma_{r r}-\sigma_{\theta \theta}\right)+\mathrm{P} \frac{\partial \omega_{2}}{\partial z}+\rho \Omega^{2} u_{r}=\rho \frac{\partial^{2} u_{r}}{\partial t^{2}} \\
\frac{\partial \tau_{r z}}{\partial r}+\frac{\partial \sigma_{z z}}{\partial z}+\frac{1}{r} \tau_{r z}+\frac{\mathrm{P}}{r} \frac{\partial\left(\mathrm{r} \omega_{2}\right)}{\partial z}+\rho \Omega^{2} u_{z}=\rho \frac{\partial^{2} u_{z}}{\partial t^{2}}
\end{gathered}
$$

where the investimal rotation vector

$\omega=\left(\omega_{1}, \omega_{2}, \omega_{3}\right)=\frac{1}{2} \operatorname{Curl}(\mathrm{u})=\left(0, \frac{1}{2}\left(\frac{\partial u_{r}}{\partial z}-u_{z}\right), \quad 0\right), \omega_{2}=\frac{1}{2}\left(\frac{\partial u_{r}}{\partial z}-u_{z}\right)$

$\mathrm{P}$ is the inatial compression, $\rho$ is the density of bones, $\sigma_{r r}, \sigma_{\theta \theta}, \sigma_{z z}, \tau_{r z}$ are the stresses, $u_{r}, u_{z}$ are the displacements components. The term of centripetal acceleration,

$$
\vec{\Omega} \times(\vec{\Omega} \times \vec{u})=\left(-\Omega^{2} u, 0,-\Omega^{2} w\right)
$$

due to time varying motion only, where

$$
\vec{\Omega}=(0, \Omega, 0), \quad \vec{u}=\left(u_{r}, 0, u_{z}\right) .
$$


The relations of stresses-displacement for homogeneous transversely isotropic bone in two dimensions are in the form:

$$
\begin{gathered}
\sigma_{r r}=\left(c_{11}+\mathrm{P}\right) \frac{\partial u_{r}}{\partial r}+\left(c_{12}+\mathrm{P}\right) \frac{u_{r}}{r}+\left(c_{13}+\mathrm{P}\right) \frac{\partial u_{z}}{\partial z} \\
\sigma_{\theta \theta}=\left(c_{12}+\mathrm{P}\right) \frac{\partial u_{r}}{\partial r}+\left(c_{11}+\mathrm{P}\right) \frac{u_{r}}{r}+\left(c_{13}+\mathrm{P}\right) \frac{\partial u_{z}}{\partial z} \\
\sigma_{z z}=c_{13} \frac{\partial u_{r}}{\partial r}+c_{13} \frac{u_{r}}{r}+c_{33} \frac{\partial u_{z}}{\partial z} \\
\tau_{r z}=c_{44}\left(\frac{\partial u_{z}}{\partial r}+\frac{\partial u_{r}}{\partial z}\right.
\end{gathered}
$$

where

$$
c_{11} ; \quad c_{12} ; \quad c_{22} ; \quad c_{13} ; \quad c_{23} ; \quad c_{44}, c_{11}=c_{22}, \quad c_{13}=c_{23}
$$

are the elastic constants of bones. Substituting from equations (2), into equations (1) we obtain:

$$
\begin{aligned}
& \frac{\partial^{2} u_{r}}{\partial r^{2}}+\frac{1}{r} \frac{\partial u_{r}}{\partial r}-\frac{1}{r^{2}} u_{r}+\frac{c_{44}}{c_{11}} \frac{\partial^{2} u_{r}}{\partial z^{2}}+\frac{c_{13}+c_{44}}{c_{11}} \frac{\partial^{2} u_{z}}{\partial r}+\frac{1}{2} \mathrm{P} \frac{\partial\left(\frac{\partial u_{r}}{\partial z}-u_{z}\right)}{\partial z}=\frac{\rho}{c_{11}}\left[\frac{\partial^{2} u_{r}}{\partial t^{2}}-\Omega^{2} u_{r}\right], \\
& \frac{\partial^{2} u_{z}}{\partial z^{2}}+\frac{c_{44}}{c_{33}}\left[\frac{\partial^{2} u_{z}}{\partial r^{2}}+\frac{1}{r} \frac{\partial u_{z}}{\partial r}\right]+\frac{c_{13}+c_{44}}{c_{33}}\left[\frac{\partial^{2} u_{r}}{\partial r \partial z}+\frac{1}{r} \frac{\partial u_{r}}{\partial z}\right]+\frac{\mathrm{P}}{2 r} \frac{\partial\left(\mathrm{r}\left(\frac{\partial u_{r}}{\partial z}-u_{z}\right)\right)}{\partial z}=\frac{\rho}{c_{33}}\left[\frac{\partial^{2} u_{z}}{\partial t^{2}}-\Omega^{2} u_{z}\right]
\end{aligned}
$$

The system (3) can be simplified as

$$
\begin{gathered}
-2\left(c_{11}-r^{2} \rho \Omega^{2}\right) u_{r}+r\left(2 c_{11} \frac{\partial u_{r}}{\partial r}+r\left(-2 \rho \frac{\partial^{2} u_{r}}{\partial t^{2}}+\mathrm{a} 1 \frac{\partial^{2} u_{r}}{\partial z^{2}}+\mathrm{a} 2 \frac{\partial^{2} u_{z}}{\partial r \partial z}+2 c_{11} \frac{\partial^{2} u_{r}}{\partial r^{2}}\right)\right)=0, \\
\frac{1}{2 r}\left(\mathrm{a} 4 \frac{\partial u_{r}}{\partial z}+\mathrm{a} 3 \frac{\partial u_{z}}{\partial r}+r\left(2 \rho \Omega^{2} \mathrm{u} 3-2 \rho \frac{\partial^{2} u_{z}}{\partial t^{2}}+2 c_{33} \frac{\partial^{2} u_{z}}{\partial z^{2}}+\mathrm{a} 4 \frac{\partial^{2} u_{r}}{\partial r \partial z}+\mathrm{a} 3 \frac{\partial^{2} u_{z}}{\partial r^{2}}\right)\right)=0,
\end{gathered}
$$

where

$$
\begin{aligned}
a_{1}=\left(c_{44}+P\right) ; \quad & a_{2}=\left(2 c_{13}+c_{44}-P\right) ; \quad a_{3}=\left(c_{44}-P\right) ; \\
a_{4}=\left(2 c_{13}+c_{44}+P\right) . &
\end{aligned}
$$

\section{Solution of the problem}

Using Helmohltz's theorem [22] the displacement vector $\overleftarrow{u}$ can be written as

$$
\overleftarrow{u}=\underline{\nabla} \Phi+\underline{\nabla} \wedge \overleftarrow{\Psi}
$$

Where the two functions $\Phi$ and $\overleftarrow{\Psi}$ are known in the theory of elasticity, by Lame' potentials irrotational and rotatinal parts of the displacement vector $\overleftarrow{u}$ respectively. The cylinder being bounded by the curved surface, therefore the stress distribution includes the effect of both $\Phi$ and $\overleftarrow{\psi}$. It is possible to take only one components of the vector $\overleftarrow{\psi}$ to be non zero as: $\overleftarrow{\psi}=(0, \psi, 0)$. From (5) we obtain:

$$
u_{r}=\frac{\partial \Phi}{\partial r}-\frac{\partial \psi}{\partial z},
$$


EFFECT OF THE INITIAL STRESS AND ROTATION ON FREE VIBRATIONS

$$
u_{z}=\frac{\psi}{r}+\frac{\partial \Phi}{\partial z}+\frac{\partial \psi}{\partial r}
$$

Substituting from equations (7) into equations (4) we get two independent equations for ? and $\psi$ as follows:

$$
\begin{gathered}
-a_{2} \frac{\partial \psi}{\partial z}+2\left(c_{11}-r^{2} \rho \Omega^{2}\right)\left(\frac{\partial \psi}{\partial z}-\frac{\partial \Phi}{\partial r}\right)+r\left(\left(a_{2}-2 c_{11}\right) \frac{\partial^{2} \psi}{\partial r \partial z}+F_{1}\right)=0 \\
\frac{1}{2 r^{3}}\left(\left(a_{3}+2 r^{2} \rho \Omega^{2}\right) \psi+r\left(-a_{3} \frac{\partial \psi}{\partial r}+r\left(-2 \rho \frac{\partial^{2} \psi}{\partial t \partial t}-\left(a_{4}-2 c_{33}\right) \frac{\partial^{2} \psi}{\partial z^{2}}+F_{2}\right)\right)\right)=0 \\
F_{1}=2 c_{11} \frac{\partial^{2} \Phi}{\partial r^{2}}+r\left(\begin{array}{c}
-a_{1} \frac{\partial^{3} \psi}{\partial z^{3}}+2 \rho\left(\frac{\partial^{3} \psi}{\partial r \partial t \partial t}-\frac{\partial^{3} \Phi}{\partial r \partial t \partial t}\right)+\left(a_{1}+a_{2}\right) \frac{\partial^{3} \Phi}{\partial r \partial z \partial z} \\
+\left(a_{2}-2 c_{11}\right) \frac{\partial^{3} \psi}{\partial r \partial r \partial z}+2 c_{11} \frac{\partial^{3} \Phi}{\partial r^{3}}
\end{array}\right) \\
F_{2}=\left(a_{3}+a_{4}\right) \frac{\partial^{2} \Phi}{\partial r \partial z}+2 a_{3} \frac{\partial^{2} \psi}{\partial r \partial r}+r\left(\begin{array}{c}
2 c_{33} \frac{\partial^{3} \Phi}{\partial z^{3}}+2 \rho\left(-\frac{\partial^{3} \Phi}{\partial r \partial t \partial t}+\Omega^{2}\left(\frac{\partial \Phi}{\partial z}+\frac{\partial \psi}{\partial \mathrm{r}}\right)-\frac{\partial^{3} \psi}{\partial r \partial t \partial t}\right) \\
-\left(a_{4}-2 c_{33}\right) \frac{\partial^{3} \psi}{\partial r \partial z \partial z}+\left(a_{3}+a_{4}\right) \frac{\partial^{3} \Phi}{\partial r \partial z}+a_{3} \frac{\partial^{3} \psi}{\partial r^{3}}
\end{array}\right)
\end{gathered}
$$

To study the propagation of harmonic waves in the $z$-direction, we assume a solution in the form:

$$
\begin{aligned}
& \Phi(r, z, t)=\Phi(r) e^{i(\gamma z-w t),} \\
& \psi(r, z, t)=\psi(r) e^{i(\gamma z-w t),}
\end{aligned}
$$

where $\gamma$ is the wave number, $\omega$ is the angular frequency. Substituting from equations. (10) into equations.(8) and (9) and omitting the factor exponential throughout, we have:

$$
\begin{gathered}
\left(a_{5}+r^{2} a_{6}\right) \Psi(\mathrm{r})+i\left(\begin{array}{c}
\left(2 c_{11}+r^{2} a_{7}\right) \Phi^{\prime}(\mathrm{r})-i r \\
\left(a_{8} \Psi^{\prime}(\mathrm{r})-2 i c_{11} \Phi^{\prime \prime}(\mathrm{r})+a_{9} r \Psi^{\prime \prime}(\mathrm{r})-2 ? c_{11} r \Phi^{(3)}(\mathrm{r})\right)
\end{array}\right)=0, \\
r^{3} a_{10} \Phi(\mathrm{r})+\left(a_{3}+a_{11} \mathrm{r}^{2}\right) \Psi[r]+r\left(\begin{array}{c}
r a_{12} \Phi^{\prime}(\mathrm{r})+\left(-a_{3}+r^{2} a_{11}\right) \Psi^{\prime}(\mathrm{r})+ \\
r\left(a_{12} r \Phi^{\prime \prime}(\mathrm{r})+a_{3}\left(2 \Psi^{\prime \prime}(\mathrm{r})+r \Psi^{(3)}(\mathrm{r})\right)\right)
\end{array}\right)=0,
\end{gathered}
$$

where

$$
\begin{array}{rlrl}
a_{5} & =\gamma\left(-a_{2}+2 c_{11}\right) ; & a_{6}=\gamma\left(a_{1} \gamma^{2}-2 \rho\left(\Omega^{2}+\omega_{5}^{2}\right)\right) ; \\
a_{7}=\left(\left(a_{1}+a_{2}\right) \gamma^{2}-2 \rho\left(\Omega^{2}+\omega_{5}^{2}\right)\right) ; & a_{8}=\left(a_{2}-2 c_{11}\right) \gamma ; \\
a_{9}=\gamma\left(a_{2}-2 c_{11}\right) ; & a_{10}=-2 i \gamma\left(\mathrm{c} 33 \gamma^{2}-\rho\left(\Omega^{2}+\omega_{5}^{2}\right)\right) ; \\
a_{11}=\left(\left(a_{4}-2 c_{33}\right) \gamma^{2}+2 \rho\left(\Omega^{2}+\omega_{5}^{2}\right)\right) ; & a_{12}=i\left(a_{3}+a_{4}\right) \gamma .
\end{array}
$$

Similar results were obtained by Dey et al. [23-24] and Elnagar. and Abd-Alla [25 ].while the first references deriving the constitutive equation for Rayleigh waves in elastic medium under initial stress, and the second reference deriving the constitutive equation for thermoelastic problems in an infinite cylinder under initial stress. From equations (11) and (12) we get equations (13) and (14) as following form

$$
\begin{gathered}
\left(a_{5}+r^{2} a_{6}\right) \Psi(\mathrm{r})+a_{8} r \Psi^{\prime}(\mathrm{r})+a_{9} i r^{2} \Psi^{\prime \prime}(\mathrm{r})=0, \\
r^{2} a_{10} \Phi(\mathrm{r})+r^{2} a_{12} \Phi^{\prime}(\mathrm{r})+a_{12} r^{2} \Phi^{\prime \prime}(\mathrm{r})=0 .
\end{gathered}
$$

Equation (13) represents the shear wave, and Equation (14) represents the longitudinal wave. The solution of equations (13) and (14) can be written in the following form:

$$
\Psi=A_{1} r^{a_{13}} J_{\mathrm{n}}\left(a_{14} r\right)+B_{1} r^{a_{13}} Y_{\mathrm{n}}\left(a_{14} r\right),
$$


EFFECT OF THE INITIAL STRESS AND ROTATION ON FREE VIBRATIONS

$$
\Phi=A_{2} J_{0}\left(a_{15} r\right)+B_{2} Y_{0}\left(a_{15} r\right),
$$

where

$$
a_{13}=\frac{1}{2}-\frac{a_{8}}{2 a_{9}} ; \quad a_{14}=\frac{\sqrt{a_{6}}}{\sqrt{a_{9}}} ; \quad a_{15}=\frac{\sqrt{a_{10}}}{\sqrt{a_{12}}} ; \quad \mathrm{n}=\frac{\sqrt{a_{8}^{2}-4 a_{5} a_{9}-2 a_{8} a_{9}+a_{9}^{2}}}{2 a_{9}},
$$

where $A_{1}, A_{2}, B_{1}$ and $B_{2}$ are arbitrary constants, $J_{\circ}$ is Bessel function of the first kind and of order zero $Y_{\circ}$ is Bessel function of the second kind and of order zero. $J_{\mathrm{n}}$ is Bessel function of the first kind and of order $\mathrm{n}, Y_{\mathrm{n}}$ is Bessel function of the second kind and of order $\mathrm{n}$. From equations (10) and (15-16) we get:

$$
\begin{gathered}
\psi(r, z, t)=e^{i(z \gamma-t \omega 5)}\left\{A_{1} r^{a_{13}} J_{\mathrm{n}}\left(a_{14} r\right)+B_{1} r^{a_{13}} Y_{\mathrm{n}}\left(a_{14} r\right)\right\}, \\
\Phi(r, z, t)=e^{i(z \gamma-t \omega 5)}\left\{A_{2} J_{0}\left(a_{15} r\right)+B_{2} Y_{0}\left(a_{15} r\right)\right\} .
\end{gathered}
$$

Substituting from eqs.(17-18) into equations $(6-7),(2)$ we obtain the final solution of the displacement components in the following form:

$$
\begin{aligned}
& u_{r}=-e^{i\left(z \gamma-t \omega_{5}\right)}\left\{A_{2} a_{15} J_{1}\left(a_{15} r\right)+B_{2} a_{15} Y_{1}\left(a_{15} r\right)+i r^{a_{13}} \gamma\left(A_{1} J_{\mathrm{n}}\left(a_{14} r\right)+B_{1} Y_{\mathrm{n}}\left(a_{14} r\right)\right)\right\}, \\
& u_{z}=e^{i\left(z \gamma-t \omega_{5}\right)}\left\{\begin{array}{l}
i A_{2} r \gamma J_{0}\left(a_{15} r\right)+A_{1} a_{14} r^{1+a_{13}} J_{\mathrm{n}-1}\left(a_{14} r\right)+A_{1}\left(1+a_{13}-n\right) r^{a_{13}} J_{\mathrm{n}}\left(a_{14} r\right) \\
+i B_{2} r \gamma Y_{0}\left(a_{15} r\right)+a_{14} B_{1} r^{1+a_{13}} Y_{\mathrm{n}-1}\left(a_{14} r\right)+B_{1}\left(1+a_{13}-n\right) r^{a} 13 Y_{\mathrm{n}}\left(a_{14} r\right)
\end{array}\right\},
\end{aligned}
$$

Substituting from equations (19-20) into equations (2) we obtain the final solution of the components stress of solid in the following form:

$$
\begin{aligned}
& \sigma_{r r}=F_{3}\left\{\begin{array}{c}
-A_{2} r\left(a_{15}^{2} c_{11}+c_{13} \gamma^{2}\right) J_{0}\left(a_{15} r\right)+a_{15} A_{2}\left(c_{11}-c_{12}\right) J_{1}\left(a_{15} r\right) \\
-i A_{1} a_{14}\left(c_{11}-c_{13}\right) r^{1+a_{13}} \gamma J_{\mathrm{n}}-1\left(a_{14} r\right)-i A_{1}\left(c_{12}-c_{13}+\left(c_{11}-c_{13}\right)\left(a_{13}-n\right)\right) \\
r^{\mathrm{a} 13} \gamma J_{\mathrm{n}}\left(a_{14} r\right)-B_{2} r\left(a_{15}^{2} c_{11}+c_{13} \gamma^{2}\right) Y_{0}\left(a_{15} r\right)+a_{15} B_{2}\left(c_{11}-c_{12}\right) \\
Y_{1}\left(a_{15} r\right)-i a_{14} B_{1}\left(c_{11}-c_{13}\right) r^{1+\mathrm{a}_{13}} \gamma Y_{\mathrm{n}-1}\left(a_{14} r\right)- \\
i B_{1}\left(c_{12}-c_{13}+\left(c_{11}-c_{13}\right)\left(a_{13}-n\right)\right) r^{a_{13}} \gamma Y_{\mathrm{n}}\left(a_{14} r\right)
\end{array}\right\}, \\
& \sigma_{\theta \theta}=F_{3}\left\{\begin{array}{c}
-A_{2} r\left(a_{15}^{2} c_{12}+c_{13} \gamma^{2}\right) J_{0}\left(a_{15} r\right)+a_{15} A_{2}\left(-c_{11}+c_{12}\right) J_{1}\left(a_{15} r\right) \\
-i A_{1} a_{14}\left(c_{12}-c_{13}\right) r^{1+a_{13}} \gamma J_{\mathrm{n}-1}\left(a_{14} r\right)-i A_{1}\left(c_{11}-c_{13}+\right. \\
\left.\left(c_{12}-c_{13}\right)\left(a_{13}-n\right)\right) r^{a_{13}} \gamma J_{\mathrm{n}}\left(a_{14} r\right)-B_{2} r\left(a_{15}^{2} c_{12}+c_{13} \gamma^{2}\right) \\
Y_{0}\left(a_{15} r\right)+a_{15} B_{2}\left(-c_{11}+c_{12}\right) Y_{1}\left(a_{15} r\right)-i a_{14} B_{1}\left(c_{12}-c_{13}\right) r^{1+a_{13}} \gamma \\
Y_{\mathrm{n}-1}\left(a_{14} r\right)-i B_{1}\left(c_{11}-c_{13}+\left(c_{12}-c_{13}\right)\left(a_{13}-n\right)\right) r^{a_{13}} \gamma Y_{\mathrm{n}}\left(a_{14} r\right)
\end{array}\right\}, \\
& \sigma_{\mathrm{zz}}=F_{3}\left\{\begin{array}{c}
-r\left(a_{15}^{2} c_{13}+c_{33} \gamma^{2}\right)\left(A_{2} J_{0}\left(a_{15} r\right)+B_{2} Y_{0}\left(a_{15} r\right)\right)-i\left(c_{13}-c_{33}\right) r^{a_{13}} \gamma \\
\left(A_{1} a_{14} r J_{\mathrm{n}-1}\left(a_{14} r\right)+A_{1}\left(1+a_{13}-n\right) J_{\mathrm{n}}\left(a_{14} r\right)+a_{14} B_{1} r Y_{\mathrm{n}-1}\left(a_{14} r\right)\right. \\
\left.+B_{1}\left(1+a_{13}-n\right) Y_{\mathrm{n}}\left(a_{14} r\right)\right)
\end{array}\right\},
\end{aligned}
$$

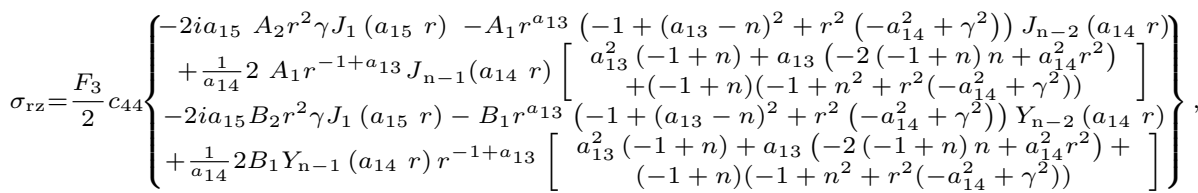

where $F_{3}=\frac{1}{r} e^{i\left(z \gamma-t \omega_{5}\right)}$ and $J_{1}$ and $Y_{1}$ is the Bessel functions of first order. In the following section, solutions of the hollow circular cylinders with three different boundary conditions are performed.

\section{Boundary conditions and frequency equation}

We are going to obtain the frequency equation for the boundary conditions. Plane vibrations cylindrical bone free surface traction, In this case, we have

$$
\sigma_{r r}(r)=\sigma_{r z}(r)=0, \quad r=a,
$$


EFFECT OF THE INITIAL STRESS AND ROTATION ON FREE VIBRATIONS IN TRANSVERSELY ISOTROPIC HUMAN LONG DRY BONE

$$
\sigma_{r r}(r)=\sigma_{r z}(r)=0 . \quad r=b .
$$

which correspond to the free inner and outer surfaces respectively. From equations. (21), (24) and (25) we obtain four homogeneous linear equations in $A_{1}, B_{1}, A_{2}$, and $B_{2}$

$$
\begin{aligned}
& -i A_{1} a_{14}\left(c_{11}-c_{13}\right) a^{1+a_{13}} \gamma J_{n-1}\left(a_{14} a\right)-i A_{1}\left(c_{12}-c_{13}+\left(c_{11}-c_{13}\right)\left(a_{13}-n\right)\right) a^{a 13} \gamma \\
& J_{n}\left(a_{14} a\right)-i a_{14} B_{1}\left(c_{11}-c_{13}\right) a^{1+a_{13}} \gamma Y_{n-1}\left(a_{14} a\right)- \\
& i B_{1}\left(c_{12}-c_{13}+\left(c_{11}-c_{13}\right)\left(a_{13}-n\right)\right) a^{a_{13}} \gamma Y_{n}\left(a_{14} a\right)- \\
& A_{2} a\left(a_{15}^{2} c_{11}+c_{13} \gamma^{2}\right) J_{0}\left(a_{15} a\right)+a_{15} A_{2}\left(c_{11}-c_{12}\right) \\
& J_{1}\left(a_{15} a\right)-B_{2} a\left(a_{15}^{2} c_{11}+c_{13} \gamma^{2}\right) Y_{0}\left(a_{15} a\right)+a_{15} B_{2}\left(c_{11}-c_{12}\right) Y_{1}\left(a_{15} a\right)=0, \\
& -i A_{1} a_{14}\left(c_{11}-c_{13}\right) a^{1+a_{13}} \gamma J_{n-1}\left(a_{14} a\right)-i A_{1}\left(c_{12}-c_{13}+\left(c_{11}-c_{13}\right)\left(a_{13}-n\right)\right) \\
& a^{a 13} \gamma J_{n}\left(a_{14} a\right)-i a_{14} B_{1}\left(c_{11}-c_{13}\right) a^{1+a_{13}} \gamma Y_{n-1}\left(a_{14} a\right)- \\
& i B_{1}\left(c_{12}-c_{13}+\left(c_{11}-c_{13}\right)\left(a_{13}-n\right)\right) a^{a} 13 \gamma Y_{n}\left(a_{14} a\right)- \\
& A_{2} a\left(a_{15}^{2} c_{11}+c_{13} \gamma^{2}\right) J_{0}\left(a_{15} a\right)+a_{15} A_{2}\left(c_{11}-c_{12}\right) J_{1}\left(a_{15} a\right) \\
& -B_{2} a\left(a_{15}^{2} c_{11}+c_{13} \gamma^{2}\right) Y_{0}\left(a_{15} a\right)+a_{15} B_{2}\left(c_{11}-c_{12}\right) Y_{1}\left(a_{15} a\right)=0 \\
& -A_{1} a^{a_{13}}\left(-1+\left(a_{13}-n\right)^{2}+a^{2}\left(-a_{14}^{2}+\gamma^{2}\right)\right) J_{n-2}\left(a_{14} a\right)+\frac{1}{a_{14}} 2 A_{1} a^{-1+a_{13}} J_{n-1}\left(a_{14} a\right) \\
& a_{13}^{2}(-1+n)+a_{13}\left(-2(-1+n) n+a_{14}^{2} a^{2}\right)+(-1+n)\left(-1+n^{2}+a^{2}\left(-a_{14}^{2}+\gamma^{2}\right)\right) \\
& -B_{1} a^{a_{13}}\left(-1+\left(a_{13}-n\right)^{2}+a^{2}\left(-a_{14}^{2}+\gamma^{2}\right)\right) Y_{n-2}\left(a_{14} a\right)+ \\
& B_{1} a^{a_{13}}\left(-1+\left(a_{13}-n\right)^{2}+a^{2}\left(-a_{14}^{2}+\gamma^{2}\right)\right) Y_{n-2}\left(a_{14} a\right)+ \\
& \frac{1}{a_{14}} 2 B_{1} Y_{n-1}\left(a_{14} a\right) a^{-1+a_{13}}\left[\begin{array}{c}
a_{13}^{2}(-1+n)+a_{13}\left(-2(-1+n) n+a_{14}^{2} a^{2}\right)+ \\
(-1+n)\left(-1+n^{2}+a^{2}\left(-a_{14}^{2}+\gamma^{2}\right)\right)
\end{array}\right] \\
& -2 i a_{15} A_{2} a^{2} \gamma J_{1}\left(a_{15} a\right)-2 i a_{15} B_{2} a^{2} \gamma J_{1}\left(a_{15} a\right)=0 \text {, } \\
& -i A_{1} a_{14}\left(c_{11}-c_{13}\right) b^{1+a_{13}} \gamma J_{n-1}\left(a_{14} b\right)-i A_{1} \\
& \left(c_{12}-c_{13}+\left(c_{11}-c_{13}\right)\left(a_{13}-n\right)\right) b^{a 13} \gamma J_{n}\left(a_{14} b\right) \\
& -i a_{14} B_{1}\left(c_{11}-c_{13}\right) b^{1+a_{13}} \gamma Y_{n-1}\left(a_{14} b\right)- \\
& i B_{1}\left(c_{12}-c_{13}+\left(c_{11}-c_{13}\right)\left(a_{13}-n\right)\right) b^{a_{13}} \gamma Y_{n}\left(a_{14} b\right) \\
& -A_{2} b\left(a_{15}^{2} c_{11}+c_{13} \gamma^{2}\right) J_{0}\left(a_{15} b\right)+a_{15} A_{2}\left(c_{11}-c_{12}\right) J_{1}\left(a_{15} b\right)- \\
& B_{2} b\left(a_{15}^{2} c_{11}+c_{13} \gamma^{2}\right) Y_{0}\left(a_{15} b\right)+a_{15} B_{2}\left(c_{11}-c_{12}\right) Y_{1}\left(a_{15} b\right)=0, \\
& -A_{1} b^{a_{13}}\left(-1+\left(a_{13}-n\right)^{2}+b^{2}\left(-a_{14}^{2}+\gamma^{2}\right)\right) J_{n-2}\left(a_{14} b\right)+\frac{1}{a_{14}} 2 A_{1} b^{-1+a_{13}} J_{n-1}\left(a_{14} b\right) \\
& {\left[a_{13}^{2}(-1+n)+a_{13}\left(-2(-1+n) n+a_{14}^{2} b^{2}\right)+(-1+n)\left(-1+n^{2}+b^{2}\left(-a_{14}^{2}+\gamma^{2}\right)\right)\right]} \\
& -B_{1} b^{a_{13}}\left(-1+\left(a_{13}-n\right)^{2}+b^{2}\left(-a_{14}^{2}+\gamma^{2}\right)\right) Y_{n-2}\left(a_{14} b\right)+\frac{1}{a_{14}} 2 B_{1} Y_{n-1}\left(a_{14} b\right) b^{-1+a_{13}} \\
& {\left[a_{13}^{2}(-1+n)+a_{13}\left(-2(-1+n) n+a_{14}^{2} b^{2}\right)+(-1+n)\left(-1+n^{2}+b^{2}\left(-a_{14}^{2}+\gamma^{2}\right)\right)\right]} \\
& -2 i a_{15} A_{2} b^{2} \gamma J_{1}\left(a_{15} b\right)-2 i a_{15} B_{2} b^{2} \gamma J_{1}\left(a_{15} b\right)=0 \text {. }
\end{aligned}
$$

These are a set of four homogeneous algebraic equations involving four unknowns integration constants $A_{1}, B_{1}, A_{2}$ and $B_{2}$. For a nontrivial solution of these equations, the determinant of the coefficient matrix must vanish. The zero determinant of the coefficient matrix will give the frequency equation for the surface waves. Thus, elimination of these unknowns would give us the frequency equation as:

$$
\Delta=\left|\begin{array}{llll}
B_{11} & B_{12} & B_{13} & B_{14} \\
B_{21} & B_{22} & B_{23} & B_{24} \\
B_{31} & B_{32} & B_{33} & B_{34} \\
B_{41} & B_{42} & B_{43} & B_{44}
\end{array}\right|=0
$$

where:

$$
\begin{aligned}
& B_{11}=-i a_{14}\left(c_{11}-c_{13}\right) a^{1+a_{13}} \gamma J_{n-1}\left(a_{14} a\right)- \\
& i\left(c_{12}-c_{13}+\left(c_{11}-c_{13}\right)\left(a_{13}-n\right)\right) a^{a 13} \gamma J_{n}\left(a_{14} a\right) ; \\
& B_{12}=-i a_{14}\left(c_{11}-c_{13}\right) a^{1+a_{13}} \gamma Y_{n-1}\left(a_{14} a\right)- \\
& i\left(c_{12}-c_{13}+\left(c_{11}-c_{13}\right)\left(a_{13}-n\right)\right) a^{a_{13}} \gamma Y_{n}\left(a_{14} a\right) ; \\
& B_{13}=-a\left(a_{15}^{2} c_{11}+c_{13} \gamma^{2}\right) J_{0}\left(a_{15} a\right)+a_{15}\left(c_{11}-c_{12}\right) J_{1}\left(a_{15} a\right) ;
\end{aligned}
$$


EFFECT OF THE INITIAL STRESS AND ROTATION ON FREE VIBRATIONS

$$
\begin{aligned}
& B_{14}=-a\left(a_{15}^{2} c_{11}+c_{13} \gamma^{2}\right) Y_{0}\left(a_{15} a\right)+a_{15}\left(c_{11}-c_{12}\right) Y_{1}\left(a_{15} a\right) \\
& B_{21}=-a^{a_{13}}\left(-1+\left(a_{13}-n\right)^{2}+a^{2}\left(-a_{14}^{2}+\gamma^{2}\right)\right) J_{n-2}\left(a_{14} a\right)+ \\
& \frac{1}{a_{14}} 2 a^{-1+a_{13}} J_{n-1}\left(a_{14} a\right)\left[\begin{array}{c}
a_{13}^{2}(-1+n)+a_{13}\left(-2(-1+n) n+a_{14}^{2} a^{2}\right) \\
+(-1+n)\left(-1+n^{2}+a^{2}\left(-a_{14}^{2}+\gamma^{2}\right)\right)
\end{array}\right] \text {; } \\
& B_{22}=-a^{a_{13}}\left(-1+\left(a_{13}-n\right)^{2}+a^{2}\left(-a_{14}^{2}+\gamma^{2}\right)\right) Y_{n-2}\left(a_{14} a\right)+ \\
& \frac{1}{a_{14}} 2 Y_{n-1}\left(a_{14} a\right) a^{-1+a_{13}}\left[\begin{array}{c}
a_{13}^{2}(-1+n)+a_{13}\left(-2(-1+n) n+a_{14}^{2} a^{2}\right) \\
+(-1+n)\left(-1+n^{2}+a^{2}\left(-a_{14}^{2}+\gamma^{2}\right)\right)
\end{array}\right] \text {; } \\
& B_{23}=-2 i a_{15} a^{2} \gamma J_{1}\left(a_{15} a\right) ; \quad B_{24}=-2 i a_{15} a^{2} \gamma J_{1}\left(a_{15} a\right) ; \\
& B_{31}=-i a_{14}\left(c_{11}-c_{13}\right) b^{1+a_{13}} \gamma J_{n-1}\left(a_{14} b\right)- \\
& i\left(c_{12}-c_{13}+\left(c_{11}-c_{13}\right)\left(a_{13}-n\right)\right) b^{a 13} \gamma J_{n}\left(a_{14} b\right) \text {; } \\
& B_{32}=-i a_{14}\left(c_{11}-c_{13}\right) b^{1+a_{13}} \gamma Y_{n-1}\left(a_{14} b\right)- \\
& i\left(c_{12}-c_{13}+\left(c_{11}-c_{13}\right)\left(a_{13}-n\right)\right) b^{a_{13}} \gamma Y_{n}\left(a_{14} b\right) ; \\
& B_{33}=-b\left(a_{15}^{2} c_{11}+c_{13} \gamma^{2}\right) J_{0}\left(a_{15} b\right)+a_{15}\left(c_{11}-c_{12}\right) J_{1}\left(a_{15} b\right) ; \\
& B_{34}=-b\left(a_{15}^{2} c_{11}+c_{13} \gamma^{2}\right) Y_{0}\left(a_{15} b\right)+a_{15}\left(c_{11}-c_{12}\right) Y_{1}\left(a_{15} b\right) ; \\
& B_{41}=-b^{a_{13}}\left(-1+\left(a_{13}-n\right)^{2}+b^{2}\left(-a_{14}^{2}+\gamma^{2}\right)\right) J_{n-2}\left(a_{14} b\right)+\frac{1}{a_{14}} 2 b^{-1+a_{13}} \\
& J_{n-1}\left(a_{14} b\right)\left[\begin{array}{c}
a_{13}^{2}(-1+n)+a_{13}\left(-2(-1+n) n+a_{14}^{2} b^{2}\right)+ \\
(-1+n)\left(-1+n^{2}+b^{2}\left(-a_{14}^{2}+\gamma^{2}\right)\right)
\end{array}\right] \\
& B_{42}=-b^{a_{13}}\left(-1+\left(a_{13}-n\right)^{2}+b^{2}\left(-a_{14}^{2}+\gamma^{2}\right)\right) Y_{n-2}\left(a_{14} b\right) \\
& +\frac{1}{a_{14}} 2 Y_{n-1}\left(a_{14} b\right) b^{-1+a_{13}}\left[\begin{array}{c}
a_{13}^{2}(-1+n)+a_{13}\left(-2(-1+n) n+a_{14}^{2} b^{2}\right) \\
+(-1+n)\left(-1+n^{2}+b^{2}\left(-a_{14}^{2}+\gamma^{2}\right)\right)
\end{array}\right] ; \\
& B_{43}=-2 i a_{15} b^{2} \gamma J_{1}\left(a_{15} b\right) \text {; } \\
& B_{44}=-2 i a_{15} b^{2} \gamma J_{1}\left(a_{15} b\right) .
\end{aligned}
$$

The roots of equation (27) gives the values of natural frequency for the free surfaces of the cylinder.

\section{Numerical results and discussion}

we investigated the frequency equations given by (27), numerically for a particular model. For the numerical calculation of frequency and phase velocity under effect of initial stress and rotation, Since these equations is an implicit function relation of natural frequency $W$, therefore one can proceed to find the variation of natural frequency with ratio $h$. Once the natural frequency is computed, the corresponding effect of initial stress and rotation. In order to study numerically the effect of initial stress and rotation on the frequency equations for natural frequency (the eigenvalues) by taking values of ratio $h$. As an illustrative purpose of the foregoing solutions, the cylinder has the following geometric and material constants which are used in the computations are given by $[1-2,14]$. $c_{11}=2.12 \times 10^{7}, c_{12}=0.95 \times 10^{7}, c_{13}=1.02 \times 10^{7}, c_{44}=0.75 \times 10^{7}, c_{33}=4.981 \times 10^{7}$, $\rho=3.986 \times 10^{2}$.For various values of frequency, the wave numbers are obtained from frequency equation flexural modes $(n=1)$. Here, we explain graphically our results of the 
EFFECT OF THE INITIAL STRESS AND ROTATION ON FREE VIBRATIONS IN TRANSVERSELY ISOTROPIC HUMAN LONG DRY BONE

previous applications. The frequencies $W$ and phase velocity were calculated with the aid of an electronic computer by using half-interval method. Fig. 1 . show the variation of phase velocity with respect to initial stress $\mathrm{P}$ for different values of rotation $\Omega=0.1,0.4,0.8$ and 1.3 for free surfaces, the phase velocity decrease with increasing the initial stress, but the phase velocity increase with increasing the values of rotation. Fig. 2. show the dimensionless frequency with respect to initial stress $\mathrm{P}$ for different values of rotation $\Omega=0.1,0.4,0.8$ and 1.3 for free surfaces, the dimensionless frequency increase with increasing the initial stress, and the dimensionless frequency increase with increasing the values of rotation. Fig.3. show variation of non-dimensional frequency versus the ratio $(h)$ of inner and outer free surfaces for different values of initial stress $P=0.2,6,1.0$ and 1.5 when rotation $\Omega=0.5$. The dimensionless frequency increase with increasing the ratio $(h)$ of inner and outer free surfaces, and the dimensionless frequency increase with increasing the values of initial stress. Fig.4. show variation of non-dimensional frequency versus the ratio $(h)$ of inner and outer free surfaces for different values of rotation $\Omega=0.1,0.4,0.8$ and 1.3 when initial stress is 2.0 , the dimensionless frequency increase with increasing the ratio $(h)$ of inner and outer free surfaces, and the dimensionless frequency increase with deincreasing the values of initial stress. It is notice that, the effects of the initial stress and the rotation on the natural frequency tends to the natural frequency is increases. These results are specific for the case considered, but other cases may have different trends because of the dependences of the results on the mechanical and constants of the material [25-35]. The results indicate that the effect of initial stress rotation is very pronouced.

\section{Conclusion}

The effect of initial stress and rotation on surface wave dispersion in bone is presented. The phase velocity and the natural frequency for this problem is obtained from the frequency equation. A numerical method has been presented for obtaining estimates of the phase velocity and the frequencies of vibration of transversely isotropic bone using half-interval method. The eigenvalues are calculated for different cases and compared with those reported in the absence of initial stress and rotation. The effects of initial stress and rotation on the frequencies and the phase velocity were indecated by figures. 
EFFECT OF THE INITIAL STRESS AND ROTATION ON FREE VIBRATIONS

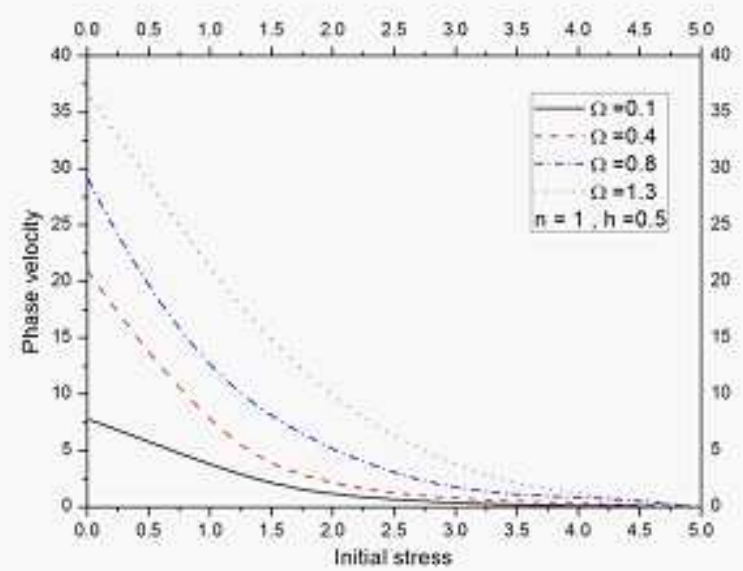

Figure 1: Variation of phase velocity with respect to initial stress $\mathrm{P}$ for diferent values of rotation $\Omega$ for inner and outer free surfaces

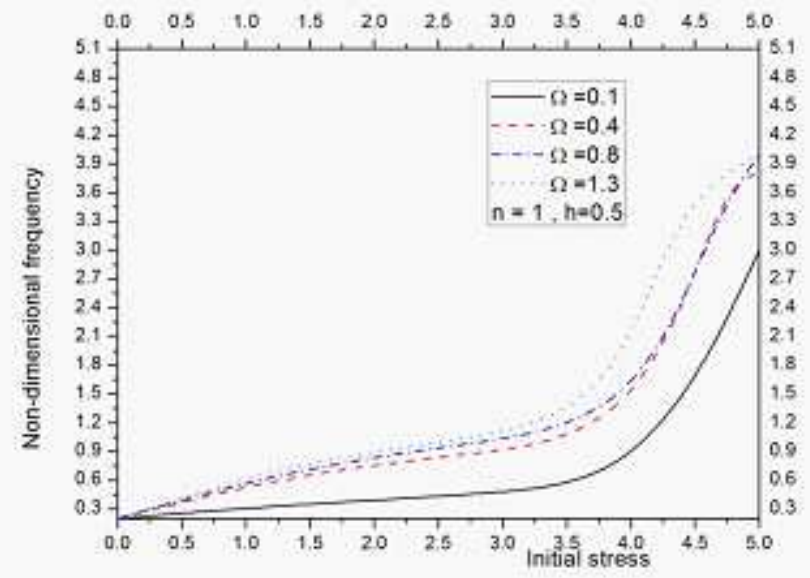

Figure 2: Variation of the dimensionless frequency with respect to initial stress for different values of rotation $\Omega$ for inner and outer free surfaces 


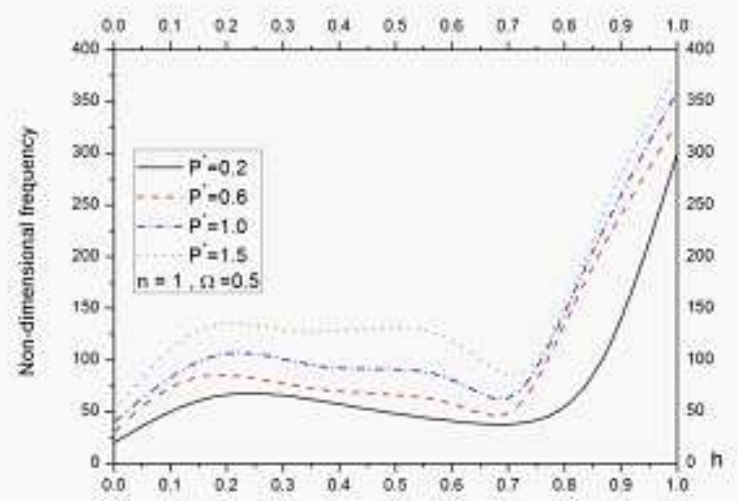

Figure 3: Variation of non-dimensional frequency versus the ratio $(h)$ of inner and outer free surfaces for for different values of initial stress when rotation $\Omega=0.5$

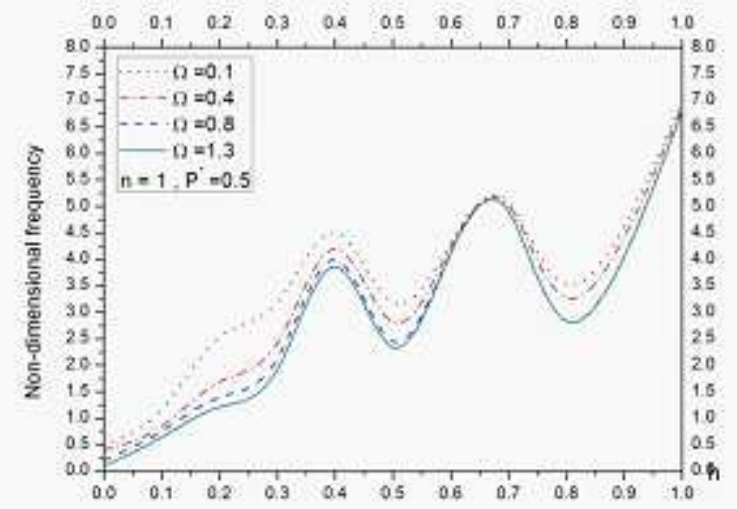

Figure 4: Variation of non-dimensional frequency versus the ratio $(h)$ of inner and outer free surfaces for for different values of rotation $\Omega$ when initial stress is 0.5 
EFFECT OF THE INITIAL STRESS AND ROTATION ON FREE VIBRATIONS

\section{References}

[1] S. R. Mahmoud, Wave propagation in cylindrical poroelastic dry bones, Applied Mathematics \& Information Sciences, Vol. 4, No.2,209-226; (2010).

[2] A. M. Abd-Alla, S. R. Mahmoud and S.M. Abo-Dahab, " Wave propagation modeling in cylindrical human long wet bones with cavity", Meccanica, Vol. 46, 6, pp.1413-1428, (2011).

[3] Salah Ramtani, Electro-mechanics of bone remodeling, International Journal of Engineering Science 46 , pp.1173-1182, (2008)

[4] S. Ramtani, M. Zidi, A theoretical model of the effect of continuum damage on a bone adaptation model, J. Biomech. 34, 471-479,(2001).

[5] S. R. Mahmoud, Influnce of rotation and generalized magneto-thermoelastic on Rayleigh waves in a granular medium under effect of initial stress and gravity field, Meccanica, Vol. 47, N0.7 pp.1561-1579, (2012).

[6] S. R. Mahmoud, Effect of rotation and magnetic field through porous medium on Peristaltic transport of a Jeffrey fluid in tube, Mathematical Problems in Engineering, Vol. 2011, ID 971456, (2011).

[7] D.P. Nickerson, N.P. Smith, P.J. Hunter, A Model of Cardiac cellular electromechanics. The integrated heart: modelling cardiac structure and function,Philos. Trans.: Math., Phys. Eng. Sci. 359, 1783 1159-1172,(2001).

[8] Peter Kohl, Frederick Sachs, Mechanoelectric feedback in cardiac cells. The integrated heart: modelling cardiac structure and function, Philos. Trans.:Math., Phys. Eng. Sci. $359,783,1173-1185,(2001)$.

[9] A.C. Eringen, Electromagnetic theory of microstretch elasticity and bone modelling, Int. J. Eng. Sci. 42, 231-242, (2004).

[10] A. M. Abd-Alla and S. R. Mahmoud, Magneto-thermoelastic problem in rotating non-homogeneous orthotropic hollow cylindrical under the hyperbolic heat conduction model, Meccanica, 45, 4, 451-462, (2010).

[11] A.M. Abd-Alla and S. R. Mahmoud, Analytical solution of wave propagation in nonhomogeneous orthotropic rotating elastic media, Journal of Mechanical Science and Technology, Vol. 26, N0. 3, pp.917-926, (2012).

[12] A. M. Abd-Alla, S. R. Mahmoud, S.M. Abo-Dahab and M.I.R.Helmi, Propagation of $S$-wave in a non-homogeneous anisotropic incompressible and initially stressed medium under influence of gravity field, Applied Mathematics and Computation, Vol. 217, 9, 4321-4332, (2011).

[13] F.Honarvarla, E.Enjilela, A.Sinclair and S.Mirnezami, Wave propagation in transversely isotropic cylinders, Int. J. Solids and Structures, Vol. 44, pp.5236-5246, (2007(.

[14] H.J.Ding , W.Q.Chen and L.Zhang, Elasticity of transversely isotropic materials, Springer, (2006).

[15] W.Q. Chen, K.Y. Lee, H. J. Ding, On free vibration of non-homogeneous transversely isotropic magneto-electro-elastic hollow cylinders, J. Sound Vib. vol. 279, 237-251 (2005)

[16] W.Q.Chen, K.Y.Lee and H.J.Ding, General solution for transversely isotropic magneto-electro-thermo-elasticity and potential theory method, I.J.Engineering Science, Vol. 42, pp. 1361-1379, (2004). 
[17] A.M. Abd-Alla, S. R. Mahmoud and S.M. Abo-Dahab, On Problem of Transient Coupled Thermoelasticity of an Annular Fin, Meccanica, Vol. 47, N0 5. pp. 1295-1306, (2012).

[18] A.M.Abd-Alla and S. R. Mahmoud, On problem of radial vibrations in nonhomogeneity isotropic cylinder under influence of initial stress and magnetic field, Journal of Vibration and Control, doi: 10.1177/1077546312441043 , (2012).

[19] M.R.Mofakhami, H.H.Toudeshky and Sh.H.Hashemi, Finite cylinder vibrations with different end boundary conditions, J. Sound and Vibration, Vol. 297, pp.293-314, (2006).

[20] A. M. Abd-Alla , G. A. Yahya, S. R. Mahmoud, H. S. Alosaimi, Effect of the rotation, magnetic field and initial stress on peristaltic motion of micropolar fluid, Meccanica, Vol. 47, N0. 6, pp.1455-1465, (2012).

[21] A.M. Abd-Alla, S. R. Mahmoud and N.A.AL-Shehri, Effect of the rotation on a nonhomogeneous infinite cylinder of orthotropic material, Applied Mathematics and Computation, Vol. 217, 22, pp 8914-8922, (2011).

[22] S. R. Mahmoud, A.M. Abd-Alla and N.A.AL-Shehri, Effect of the rotation on plane vibrations in a transversely isotropic infinite hollow cylinder, International Journal of Modern Physics B, Vol. 25,26, pp. 3513-3528 (2011).

[23] M. Marin, G. Stan, Weak solutions in Elasticity of dipolar bodies with stretch, Carpathian Journal of Mathematics, Vol. 29 (1), 2013, pp. 33-40

[24] K. Sharma, M. Marin, Reflection and transmission of waves from imperfect boundary between two heat conducting micropolar thermoelastic solids, An. Sti. Univ. Ovidius Constanta, Vol. 22, issue 2,(2014), 151-175

[25] A.M.El-Naggar and A.M.Abd-Alla, On a generalized thermo-elastic problems in an infinite cylinder under initial stress, J. Earth, Moon and Planets, Vol. 37, PP.213223, (1987).

[26] Haixia Lu, Li Sun, Jingxian Sun, Existence of positive solutions to a non-positive elastic beam equation with both ends fixed, Boundary Value Problems 2012, 2012:56 (2012).

[27] S. R. Mahmoud, Effect of non-homogeneity and rotation on an infinite generalized thermoelastic diffusion medium with a spherical cavity subject to magnetic field and initial stress, Abstract and Applied Analysis, Vol. 2013, Article ID 284646, in press, (2013).

[28] M. Marin, Some estimates on vibrations in thermoelasticity of dipolar bodies, Journal of Vibration and Control, 2010, 16 (1), 33-47 .

[29] S. R. Mahmoud, Effect of non-homogenity, magnetic field and gravity field on Rayleigh waves in an initially stressed elastic half-space of orthotropic material subject to rotation, Journal of Computational and Theoretical Nanoscience, Vol.11, pp. 1627$1634,(2014)$.

[30] M. Marin, The Lagrange identity method in thermoelasticity of bodies with microstructure, Int. J. Engng. Sci., Elsevier, vol. 32, 8, 1229-1240; (1994).

[31] M. Marin, A partition of energy in Thermoelasticity of microstretch bodies, Nonlinear Analysis: R. W. A., Elsevier, vol. 11, 4, 2436-2447; (2010).

[32] S. R. Mahmoud, Analytical solution for free vibrations of elastodynamic orthotropic hollow sphere under the influence of rotation, Journal of Computational and Theoretical Nanoscience, Vol. 11,1,pp. 137-146,(2014). 
EFFECT OF THE INITIAL STRESS AND ROTATION ON FREE VIBRATIONS

[33] M. Marin, Ravi P Agarwal, S.R. Mahmoud, Nonsimple material problems addressed by the Lagrange's identity, Boundary Value Problems, 135, 2013:135, (2013).

[34] S. R. Mahmoud, On problem of Shear waves in a magneto-elastic half-space of initially stressed a non-homogeneous anisotropic material under influence of rotation, International Journal of Mechanical Sciences, Vol. 77, 12, pp.269-276, (2013).

[35] M. Marin, R. P. Agarwal, S. R. Mahmoud, Modeling a microstretch thermoelastic body with two temperatures, Abstract and Applied Analysis, doi.org/10.1155/2013/583464, Vol. 2013, Article ID 583464, 7 pages, (2013).

[36] M. Marin, O. Florea, On temporal behavior of solutions in Thermoelasticity of porous micropolar bodies, An. Sti. Univ. Ovidius Constanta, Vol. 22, issue 1,(2014), 169-188

[37] X. Lin, B. Zhao and Z. Du, A third-order multi-point boundary value problem at resonance with one three dimensional kernel space, Carpathian Journal of Mathematics, Vol. 30 (2014), No. 1, 93-100

S. R. MAHMOUD

Science Faculty, King Abdulaziz University,Saudi Arabia

Science Faculty, Sohag University, Egypt

P.O BOX 80203 JEDDAH 21589,Saudi Arabia.

E-mail1: srhassan@kau.edu.sa

E-mail2: samsam73@yahoo.com

Marin MARIN,

Department of Mathematics,

Transilvania University of Braşov,

Bdul Iuliu Maniu, nr. 50, Braşov, Romania.

Email: m.marin@unitbv.ro

K.S.AL-BASYOUNI,

Department of Mathematics,

Science Faculty, King Abdulaziz University,Saudi Arabia. 